\title{
On the Robustness of Lattice-Reduction Aided Detectors in Correlated MIMO Systems
}

\author{
Dirk Wübben, Volker Kühn, and Karl-Dirk Kammeyer \\ Department of Communications Engineering, University of Bremen \\ Otto-Hahn-Allee, D-28359 Bremen, Germany \\ Phone: +49 421218 2545, Email: \{wuebben, kuehn, kammeyer\}@ant.uni-bremen.de
}

\begin{abstract}
Recently the use of lattice-reduction for signal detection in multiple antenna systems has been proposed. In combination with simple successive interference cancellation this scheme achieves near maximum-likelihood performance. To this end, the given MIMO channel is transformed into an almost orthogonal matrix leading to less noise enhancement within the detection. In this paper, we investigate the performance of common and lattice-reduction-aided detection schemes for correlated fading channels. We show, that the new scheme achieves significant gain in comparison to common algorithms. Thus, the new algorithm clearly outperforms existing methods with comparable complexity and is also more robust with respect to spatial correlation.
\end{abstract}

Index Terms-MIMO systems, BLAST, lattice-reduction, wireless communication, spatial correlation.

\section{INTRODUCTION}

Within the V-BLAST architecture parallel data streams are transmitted over $n_{T}$ different antennas in order to increase the spectral efficiency. Besides linear detection schemes based on the zero-forcing (ZF) or the minimum mean square error (MMSE) criterion, successive interference cancellation (SIC) is a popular way to detect the transmitted signals at the receiver site. Unfortunately, for ill-conditioned channel matrices all these schemes are clearly inferior to maximum-likelihood (ML) detection. However, in spatial correlated environments the probability of ill-conditioned matrices increases, resulting in an even higher error rate. Recently, lattice-reduction (LR) has been proposed in order to transform the system model into an equivalent one with better conditioned channel matrix prior to low-complexity linear or SIC detection, e.g. [1]-[3]. The extension of the LR-aided detection schemes to the MMSE criterion and a lattice-reduction algorithm with reduced complexity was presented in [4], [5]. In this work we investigate the performance of common and LR-aided detection schemes with respect to spatial correlated channels. As the performance of a MIMO system can significantly degrade when correlation is present, it is of fundamental importance to investigate this general behavior of the detection algorithms.

The remainder of this paper ist organized as follows. In Section II, the system model and notation is introduced. The basics about lattice-reduction and lattice-reduction based detection of MIMO systems are explained in Section III and in Section IV, respectively. The performance for uncorrelated and correlated channels is than investigated in Section V. Concluding remarks can be found in Section VI.

\section{SYSTEM DESCRIPTION}

We consider the multiple antenna system shown in Fig. 1 with $n_{T}$ transmit and $n_{R} \geq n_{T}$ receive antennas in a flat fading environment. According to the V-BLAST architecture, the data is demultiplexed into $n_{T}$ data substreams of equal length. These substreams are mapped onto $M$-QAM symbols and transmitted over the $n_{T}$ antennas simultaneously.

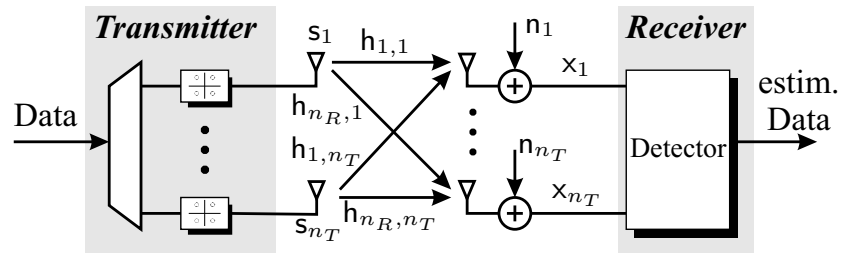

Fig. 1. Model of a MIMO system with $n_{T}$ transmit and $n_{R}$ receive antennas.

For the mathematical description of the MIMO system we investigate one time slot of the time-discrete complex baseband model. Let ${ }^{1} \mathbf{s}$ denote the complex valued $n_{T} \times 1$ transmit signal vector, then the corresponding $n_{R} \times 1$ receive signal vector $\mathbf{x}$ is given by

$$
\mathbf{x}=\mathbf{H s}+\mathbf{n} .
$$

In (1), $\mathbf{n}$ represents white gaussian noise of variance $\sigma_{n}^{2}$ observed at the $n_{R}$ receive antennas while the average transmit power of each antenna is normalized to one, i.e. $\mathrm{E}\left\{\mathbf{s s}^{H}\right\}=$ $\mathbf{I}_{n_{T}}$ and $\mathrm{E}\left\{\mathbf{n n}^{H}\right\}=\sigma_{n}^{2} \mathbf{I}_{n_{R}}$. We assume a flat fading environment, where the $n_{R} \times n_{T}$ channel matrix $\mathbf{H}$ is constant over a frame and changes independently from frame to frame (block fading channel). In order to simulate the transmission over correlated MIMO channels we apply the popular Kronecker model [6]

$$
\mathbf{H}=\boldsymbol{\Phi}_{\mathrm{R}}^{\frac{1}{2}} \mathbf{G} \boldsymbol{\Phi}_{\mathrm{T}}^{\frac{1}{2}}
$$

with G consisting of uncorrelated complex gaussian coefficients $\mathrm{g}_{i, j}$ of unit variance. According to the correlation model presented in [7], the spatial correlation matrix at the transmitter $\boldsymbol{\Phi}_{\mathrm{T}}=\mathrm{E}\left\{\mathbf{H}^{H} \mathbf{H}\right\}$ and at the receiver $\boldsymbol{\Phi}_{\mathrm{R}}=\mathrm{E}\left\{\mathbf{H H}^{H}\right\}$ can be modelled as a function of the correlation coefficients $0 \leq$

\footnotetext{
${ }^{1}$ Throughout this paper, $(\cdot)^{T}$ and $(\cdot)^{H}$ denote matrix transpose and hermitian transpose, respectively. Furthermore, $\mathbf{I}_{\alpha}$ indicates the $\alpha \times \alpha$ identity matrix and $\mathbf{0}_{\alpha, \beta}$ denotes the $\alpha \times \beta$ all zero matrix. With $\mathcal{R}\{\cdot\}$ and $\mathcal{I}\{\cdot\}$ we denote the real part and the imaginary part, respectively.
} 
$\rho_{\mathrm{T}}, \rho_{\mathrm{R}} \leq 1$. Using their definition, the $n_{T} \times n_{T}$ correlation matrix at the transmitter is given by the Toeplitz matrix

$$
\boldsymbol{\Phi}_{\mathrm{T}}=\left[\begin{array}{ccccc}
1 & \rho_{\mathrm{T}} & \rho_{\mathrm{T}}^{4} & \cdots & \rho_{\mathrm{T}}^{\left(n_{T}-1\right)^{2}} \\
\rho_{\mathrm{T}} & 1 & \rho_{\mathrm{T}} & \cdots & \vdots \\
\vdots & \ddots & \ddots & \ddots & \rho_{\mathrm{T}} \\
\rho_{\mathrm{T}}^{\left(n_{T}-1\right)^{2}} & \cdots & \rho_{\mathrm{T}}^{4} & \rho_{\mathrm{T}} & 1
\end{array}\right]
$$

and a corresponding definition holds for the $n_{R} \times n_{R}$ matrix $\boldsymbol{\Phi}_{\mathrm{R}}$ with coefficient $\rho_{\mathrm{R}}$. The correlation model can further simplified by assuming $\rho_{\mathrm{T}}=\rho_{\mathrm{R}}=\rho$, yielding a single parameter model [7]. The given model can range form the uncorrelated case $(\rho=0.0)$ to the fully correlated scenario $(\rho=1.0)$. However, as the investigation of the MIMO detectors does not take the used correlation model into account, the general statements can also be adopted to other models. Within this paper we assume perfect knowledge about the channel state information (CSI) by the receiver, but no knowledge for the transmitter.

Treating real and imaginary parts of (1) separately, the system model can be rewritten as

$$
\mathbf{x}=\mathbf{H s}+\mathbf{n}
$$

with the real-valued channel matrix

$$
\mathbf{H}=\left[\begin{array}{cr}
\mathcal{R}\{\mathbf{H}\} & -\mathcal{I}\{\mathbf{H}\} \\
\mathcal{I}\{\mathbf{H}\} & \mathcal{R}\{\mathbf{H}\}
\end{array}\right] \in \mathbb{R}^{n \times m}
$$

and the real-valued vectors

$$
\mathbf{x}=\left[\begin{array}{c}
\mathcal{R}\{\mathbf{x}\} \\
\mathcal{I}\{\mathbf{x}\}
\end{array}\right] \quad \mathbf{s}=\left[\begin{array}{c}
\mathcal{R}\{\mathbf{s}\} \\
\mathcal{I}\{\mathbf{s}\}
\end{array}\right] \quad \mathbf{n}=\left[\begin{array}{c}
\mathcal{R}\{\mathbf{n}\} \\
\mathcal{I}\{\mathbf{n}\}
\end{array}\right] .
$$

By defining $m=2 n_{T}$ and $n=2 n_{R}$ the dimension of the real channel matrix (5) is given by $n \times m=2 n_{R} \times 2 n_{T}$. Likewise the dimension of the vectors (6) are given by $\mathrm{x} \in$ $\mathbb{R}^{n}, \mathbf{n} \in \mathbb{R}^{n}$ and $\mathbf{s} \in \mathcal{A}^{m}$, where $\mathcal{A}$ denotes the finite set of real-valued transmit signals [4]. In the sequel we will apply this real-valued representation, as we can now interpret each noiseless receive signal as a point of a lattice spanned by $\mathbf{H}$. Additionally, the performance of successive algorithms like the V-BLAST detection can be improved by separating the real and imaginary part of each transmit signal.

The optimum maximum-likelihood (ML) detector searches over the whole set of transmit signals $\mathbf{s} \in \mathcal{A}^{m}$, and decides in favor of the transmit signal $\hat{\mathbf{s}}_{\mathrm{ML}}$ wich results in the minimum euclidian distance to the receive vector $\mathbf{x}$, i.e.

$$
\hat{\mathbf{s}}_{\mathrm{ML}}=\arg \min _{\mathbf{s} \in \mathcal{A}^{m}}\|\mathbf{x}-\mathbf{H} \mathbf{s}\|^{2} \text {. }
$$

As the computational effort is of order $M^{n_{T}}$, brute force ML detection is not feasible for larger number of transmit antennas or higher modulation schemes. A feasible alternative is the application of sphere detector (SD) [8]-[11], which restricts the search space to a sphere. However, the computational complexity is still high in comparison to simple but suboptimal SIC. In the sequel, we apply lattice-reduction in order to improve the performance of SIC and linear detection. One advantage of this strategy is, that the computational overhead caused by the lattice-reduction algorithm is only required once for each transmitted frame, so for large frame length the effort for each signal vector is very small.

\section{LATTICE-REDUCTION (LR)}

By interpreting the columns $\mathbf{h}_{\ell}(1 \leq \ell \leq m)$ of the realvalued channel matrix $\mathbf{H}$ as the basis and assuming an infinite $m$ dimensional integer transmit signal space $\mathbb{Z}^{m}$, the set of all possible undisturbed receive signals constructs the lattice $\mathcal{L}(\mathbf{H})=\mathbf{H} \mathbb{Z}^{m}$. However, the same lattice $\mathcal{L}(\tilde{\mathbf{H}})=\mathcal{L}(\mathbf{H})$ is also generated by each matrix $\tilde{\mathbf{H}}=\mathbf{H T}$, as long as the $m \times m$ transformation matrix $\mathbf{T}$ is unimodular, i.e. $\mathbf{T}$ contains only integer entries and the determinant is $\operatorname{det}(\mathbf{T})= \pm 1$ [12]. As both matrices describe exactly the same receive signal space, we may chose that basis which offers nicer properties for the detection of MIMO systems [4].

The aim of lattice-reduction is to transform a given basis $\mathbf{H}$ into a new basis $\tilde{\mathbf{H}}$ with vectors of shortest length or, equivalently, into a basis consisting of roughly orthogonal basis vectors. Usually, $\tilde{\mathbf{H}}$ is much better conditioned than $\mathbf{H}$ and therefore leads to less noise enhancement for linear detection. In order to describe the impact of $\mathbf{H}$ on the noise enhancement, we introduce the condition number $\kappa(\mathbf{H})=\sigma_{\max } / \sigma_{\min } \geq 1$, with $\sigma_{\max }$ and $\sigma_{\min }$ denoting the largest and the smallest singular value of $\mathbf{H}$, respectively. With $\|\mathbf{H}\|_{2}$ defining the spectral norm of $\mathbf{H}$, the condition number corresponds to $\kappa(\mathbf{H})=\|\mathbf{H}\|_{2}\left\|\mathbf{H}^{-1}\right\|_{2}$. For ill-conditioned matrices $\kappa(\mathbf{H})$ is large, whereas for orthogonal matrices $\kappa(\mathbf{H})=1$, as no noise enhancement is caused. As the reduced basis $\tilde{\mathbf{H}}$ is chosen to have roughly orthogonal basis vectors, the corresponding condition number $\kappa(\tilde{\mathbf{H}})$ is usually much smaller than $\kappa(\mathbf{H})$. Consequently, a linear detector with respect to this new basis may achieve better performance results, as the impact of noise enhancement is reduced.

For further investigation, we introduce the QR decomposition $\mathbf{H}=\mathbf{Q R}$ with the $n \times m$ matrix $\mathbf{Q}$ having orthogonal columns of unit length $\left(\mathbf{Q}^{T} \mathbf{Q}=\mathbf{I}_{m}\right)$ and the upper triangular matrix $\mathbf{R}=\left(r_{i, j}\right)_{1 \leq i, j \leq m}$. In the same way, the decomposition $\tilde{\mathbf{H}}=\tilde{\mathbf{Q}} \tilde{\mathbf{R}}$ is defined. With respect to this QR decomposition, the basis vector $\tilde{\mathbf{h}}_{\ell}$ is almost orthogonal to the space spanned by $\tilde{\mathbf{h}}_{1}, \ldots, \tilde{\mathbf{h}}_{\ell-1}$, if the elements $\left|\tilde{r}_{1, \ell}\right|, \ldots,\left|\tilde{r}_{\ell-1, \ell}\right|$ are close to zero. An efficient (though not optimal) way to determine a reduced basis was proposed by Lenstra, Lenstra and Lovàsz [12] called LLL algorithm. Starting with $\mathbf{H}=\mathbf{Q R}$ this iterative algorithm achieves a reduced basis $\tilde{\mathbf{H}}=\tilde{\mathbf{Q}} \tilde{\mathbf{R}}$ and the according transformation matrix $\mathbf{T}$. The beneficial impact of applying the Sorted QR Decomposition (SQRD) [13] to the computational complexity of the LLL algorithm was presented in [4], [5].

In order to investigate the influence of the spatial correlation on the condition of the MIMO channel, Fig. 2 shows the cumulative distribution function $(\mathrm{CDF})$ of $\kappa(\mathbf{H})$ for varying correlation coefficients $\rho$. As expected the CDFs indicate an increasing probability of large condition numbers for increasing correlation coefficients. As an ill-conditioned matrix leads to strong noise enhancement, the performance of common MIMO 


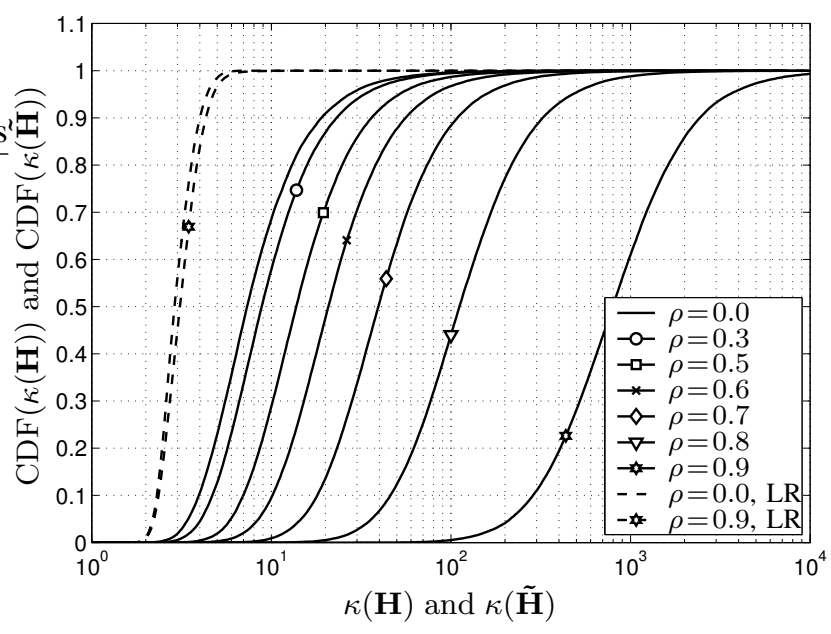

Fig. 2. Cumulative distribution function (CDF) of $\kappa(\mathbf{H})$ (solid lines) and $\kappa(\tilde{\mathbf{H}})$ (dashed lines) for various correlations coefficients $\rho$ for a system with $n_{T}=n_{R}=4$ antennas.

detectors degenerates in correlated environments. However, by investigating the distribution of the condition number $\kappa(\tilde{\mathbf{H}})$ of the lattice-reduced channel matrix $\tilde{\mathbf{H}}$, we observe almost the same CDF in case of uncorrelated and strong correlated $(\rho=0.9)$ channels. Thus, the LR-aided detection schemes introduced in the next section are supposed to be more robust with respect to correlation.

\section{LR-Aided Detection Algorithms}

\section{A. Lattice-Reduction aided Linear Detection}

By using the lattice-reduced channel matrix $\tilde{\mathbf{H}}=\mathbf{H T}$ and introducing $\mathbf{z}=\mathbf{T}^{-1} \mathbf{S}$ the receive vector (4) can be rewritten as

$$
\mathbf{x}=\mathbf{H} \mathbf{s}+\mathbf{n}=\mathbf{H T T}^{-1} \mathbf{s}+\mathbf{n}=\tilde{\mathbf{H}} \mathbf{z}+\mathbf{n} .
$$

Note that $\mathbf{H s}$ and $\tilde{\mathbf{H}} \mathbf{z}$ describe the same point in a lattice, but the LLL-reduced matrix $\tilde{\mathbf{H}}$ is usually much better conditioned than the original channel matrix $\mathbf{H}$. For $\mathbf{s} \in \mathbb{Z}^{m}$ we also have $\mathbf{z} \in \mathbb{Z}^{m}$, so $\mathbf{s}$ and $\mathbf{z}$ stem from the same set. However, for $M$-QAM, i.e. $\mathrm{s} \in \mathcal{A}^{m}$, the lattice is finite and the domain of $\mathbf{z}$ differs from $\mathcal{A}^{m}$.

The idea behind LR-aided detection is to consider the equivalent system model in (8) and perform the detection with respect to $\mathbf{z}$ instead of $\mathbf{s}$. For LR-aided $\mathrm{ZF}$ linear detection (LD) this means that first $\tilde{\mathbf{z}}_{\mathrm{LR}-\mathrm{ZF}}=\tilde{\mathbf{H}}^{+} \mathbf{X}$ is calculated, where the multiplication with the pseudo-inverse $\tilde{\mathbf{H}}^{+}=\left(\tilde{\mathbf{H}}^{T} \tilde{\mathbf{H}}\right)^{-1} \tilde{\mathbf{H}}$ usually causes less noise amplification than the multiplication with $\mathbf{H}^{+}$due to the roughly orthogonal columns of $\tilde{\mathbf{H}}$. Therefore, a hard decision based on $\tilde{\mathbf{z}}_{\mathrm{LR}-\mathrm{ZF}}$ is in general more reliable than one on $\tilde{\mathbf{s}}_{\mathrm{ZF}}=\mathbf{H}^{+} \mathbf{x}$. However, as the elements of $\mathbf{z}$ are not independent of each other, a vector quantization would be necessary [4]. A straightforward (though suboptimal) solution is to perform an unconstrained element-wise quantization $\hat{\mathbf{z}}_{\mathrm{LR}-\mathrm{ZF}}=\mathcal{Q}\left\{\tilde{\mathbf{z}}_{\mathrm{LR}-\mathrm{ZF}}\right\}$, calculate $\hat{\mathbf{s}}_{\mathrm{LR}-\mathrm{ZF}}=\mathbf{T} \hat{\mathbf{z}}_{\mathrm{LR}-\mathrm{ZF}}$, and finally restrict this result to the set $\mathcal{A}^{m}$. The principle block diagram for a LR-aided detector is shown in Fig. 3

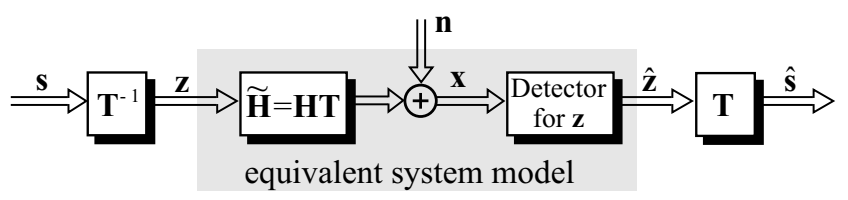

Fig. 3. Block diagram of a Lattice-Reduction based detector.

In order to improve the estimate for $\mathbf{z}$, it is meaningful to implement the LR with respect to the MMSE criterion. To this end, the LR is performed with respect to the extended channel matrix ${ }^{2} \underline{\mathbf{H}}=\left[\mathbf{H}^{T} \sigma_{n} \mathbf{I}_{m}\right]^{T}$ yielding $\underline{\tilde{\mathbf{H}}}=\underline{\mathbf{H}} \underline{\mathbf{T}}$ and compute $\tilde{\mathbf{z}}_{\mathrm{LR}-\mathrm{MMSE}}=\underline{\tilde{\mathbf{H}}}^{+} \underline{\mathbf{x}}$. As shown in [4], [5] this MMSE solution yields a significant improvement in comparison to LRaided $\mathrm{ZF}$ detectors and is also more meaningful than a MMSE solution of the LR-system model in (8).

\section{B. Lattice-Reduction aided SIC}

As $\tilde{\mathbf{H}}$ is only roughly orthogonal, the mutual influence of the transformed signals $z_{i}$ is small, but still present. Thus, a successive interference cancellation (SIC) may result in additional improvements. Applying the QR decomposition of the reduced channel matrix to the system model from (8) we get

$$
\tilde{\mathbf{z}}_{\mathrm{LR}-\mathrm{ZF}-\mathrm{SIC}}=\tilde{\mathbf{Q}}^{T} \mathbf{x}=\tilde{\mathbf{R}} \mathbf{z}+\tilde{\mathbf{Q}}^{T} \mathbf{n},
$$

where $\tilde{\mathbf{Q}}$ and $\tilde{\mathbf{R}}$ have already been calculated by the LLL algorithm. Due to the upper triangular structure of $\tilde{\mathbf{R}}$, the $m$-th element of $\tilde{\mathbf{z}}$ is free of interference and can be used to estimate $z_{m}$. Proceeding with $\tilde{z}_{m-1}, \ldots, \tilde{z}_{1}$ and assuming correct previous decisions, the interference can be perfectly cancelled in each step. It is well known, that because of error propagation the order of detection has a large influence on the performance of SIC. The optimum order can be calculated efficiently by the so-called Post-Sorting-Algorithm (PSA) proposed in [13], which exploits the fact, that the mean error in each detection step is proportional to the diagonal elements of $\tilde{\mathbf{R}}^{-1}$.

Similar to linear detection, we can consider the latticereduced version of the extended system model with the equivalent channel matrix $\underline{\tilde{\mathbf{H}}}=\tilde{\tilde{\mathbf{Q}}} \underline{\tilde{\mathbf{R}}}$. This leads to LR-aided MMSESIC with decision variables given by

$$
\tilde{\mathbf{z}}_{\mathrm{LR}-\mathrm{MMSE}-\mathrm{SIC}}=\underline{\tilde{\mathbf{Q}}}^{T} \underline{\mathbf{x}}=\underline{\tilde{\mathbf{R}}} \mathbf{z}+\boldsymbol{\eta},
$$

where the newly defined noise term $\boldsymbol{\eta}$ also incorporates residual interference. The detection procedure equals that of LR-aided ZF-SIC.

\footnotetext{
${ }^{2}$ As shown in [13], a common linear MMSE detector corresponds to a linear ZF detector with respect to the extended system model given by the $(n+m) \times m$ extended channel matrix $\underline{\mathbf{H}}$ and the $(n+m) \times 1$ extended receive vector $\underline{\mathbf{x}}$

$$
\underline{\mathbf{H}}=\left[\begin{array}{c}
\mathbf{H} \\
\sigma_{n} \mathbf{I}_{m}
\end{array}\right] \quad \text { and } \quad \underline{\mathbf{x}}=\left[\begin{array}{c}
\mathbf{x} \\
\mathbf{0}_{m, 1}
\end{array}\right] .
$$

Thus, the output of the MMSE filter can be written as $\tilde{\mathbf{s}}_{\mathrm{MMSE}}=$ $\left(\mathbf{H}^{T} \mathbf{H}+\sigma_{n}^{2} \mathbf{I}_{m}\right)^{-1} \mathbf{H}^{T} \mathbf{x}=\underline{\mathbf{H}}^{+} \underline{\mathbf{x}}$, which exactly matches the structure
} of a linear ZF detector. 


\section{Performance Analysis}

In the sequel, we investigate a MIMO system with $n_{T}=4$ transmit and $n_{R}=4$ receive antennas and 4-QAM modulation. $E_{b}$ denotes the average energy per information bit arriving at the receiver. a) Linear Detection

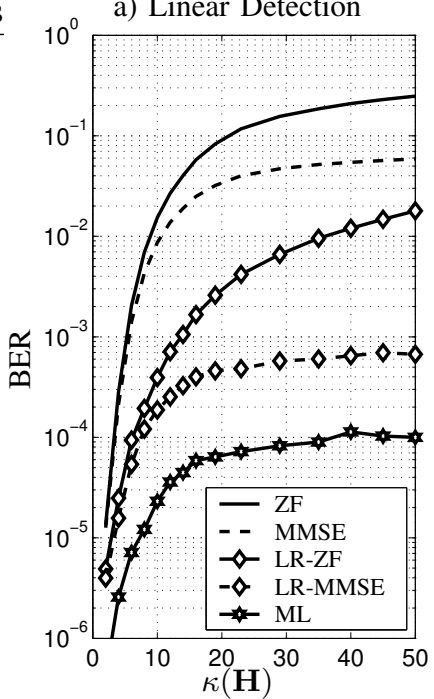

b) SIC Detection

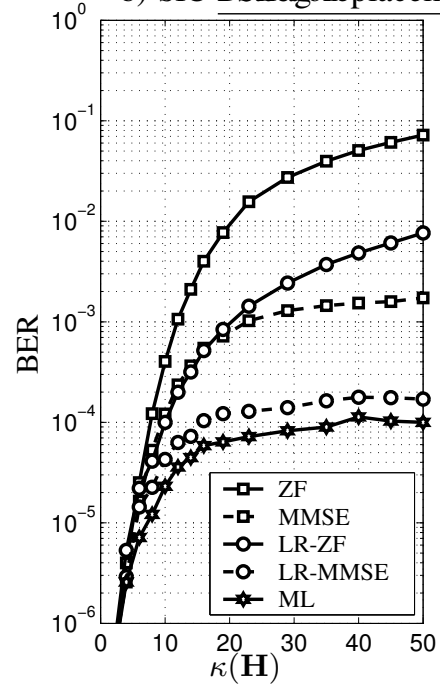

Fig. 4. BER versus condition number $\kappa(\mathbf{H})$ for a system with $n_{T}=4$ and $n_{R}=4$ antennas, 4-QAM symbols, $E_{b} / N_{0}=16 \mathrm{~dB}, \mathrm{ZF}$ (continuous lines) and MMSE (dashed lines) a) linear detection and b) SIC detection.

Fig. 4 shows the achieved BER performance of several detection schemes versus the condition number $\kappa(\mathbf{H})$ of the (uncorrelated) channel matrix and $E_{b} / N_{0}=16 \mathrm{~dB}$. Part a) contains the simulation results for the common and LR-aided linear detection schemes and ML detection. For $\kappa(\mathbf{H}) \approx 1$ all schemes achieve very good performance. However, if the matrix is ill-condition, the performances of the common $\mathrm{ZF}$ and MMSE linear detector are poor, whereas the LR-aided linear detectors achieve suitable results. The corresponding BERs for common SIC and LR-aided SIC are given in part b) of Fig. 4. The SIC schemes generally outperform the linear schemes. Thus, we will consider only SIC detection in the following investigations. Furthermore, LR-MMSE-SIC achieves almost ML performance for the whole range of investigated condition numbers.

The performance of common and LR-aided SIC versus the correlation coefficient $\rho$ is investigated in Fig. 5 for $E_{b} / N_{0}=$ $16 \mathrm{~dB}$. As the probability of large condition numbers increases with $\rho$ and the BER performance degrades with the condition number, we observe an increase of the BER with stronger correlation. Obviously, the detection with respect to the MMSE criterion clearly outperforms the corresponding ZF solutions. Again, the LR-aided MMSE-SIC achieves almost the performance of ML detection for the whole range of correlation coefficients and achieves significant gain in comparison to the common MMSE-SIC.

After investigating the dependence of the BER on the condition number and the correlation coefficient for a fixed

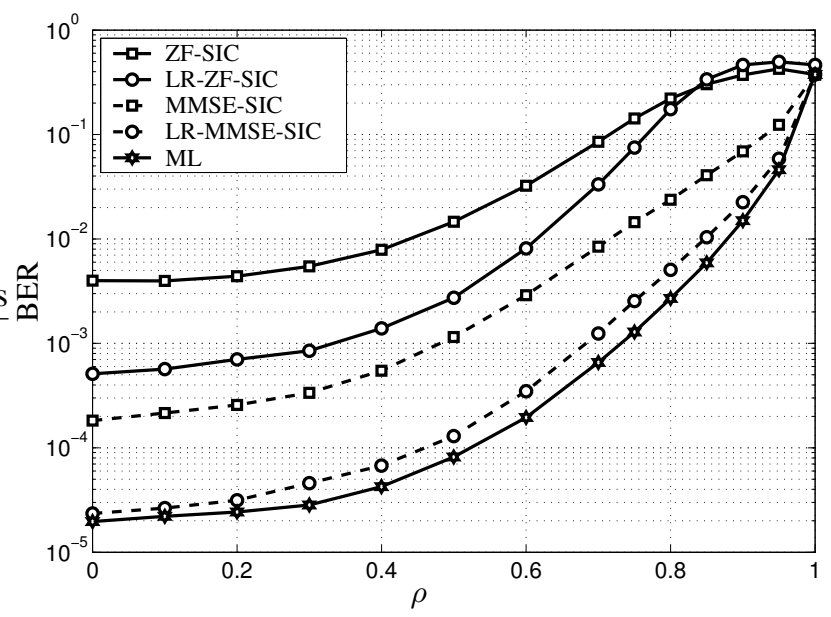

Fig. 5. BER of common SIC, LR-aided SIC and ML versus correlation coefficient $\rho$ and a system with $n_{T}=n_{R}=4$ antennas, 4-QAM modulation for $16 \mathrm{~dB}$.

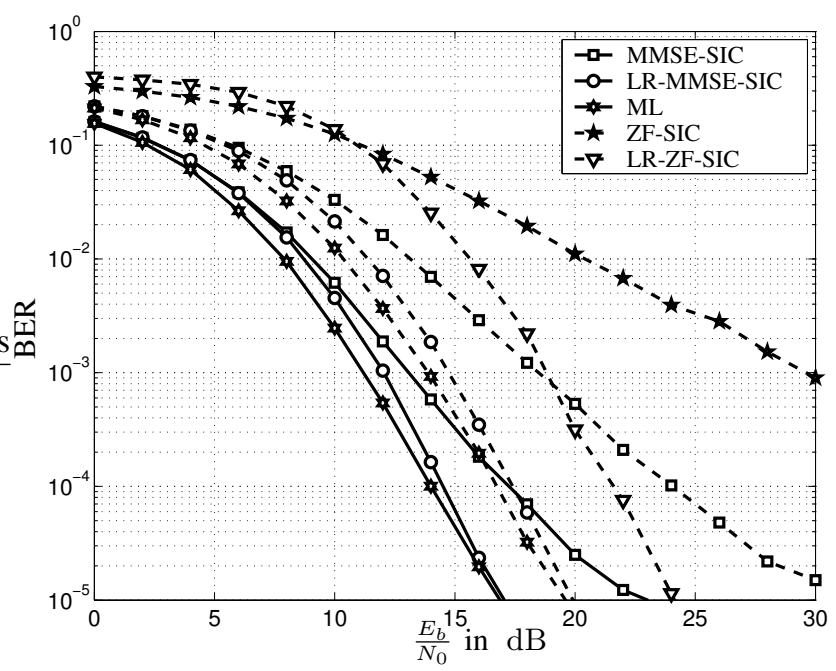

Fig. 6. BER of common and LR-aided SIC in a uncorrelated (continuous lines, $\rho=0.0$ ) and correlated (dashed lines, $\rho=0.6$ ) system with $n_{T}=$ $n_{R}=4$ antennas and 4-QAM modulation.

SNR, we will now investigate the performance versus $E_{b} / N_{0}$. Fig. 6 shows the performance of common and LR-aided MMSE-SIC for $\rho=0.0$ and $\rho=0.6$. Due to visualization reason the BERs of ZF-SIC are only included for the correlated channel. In case of uncorrelated channels, LR-MMSE-SIC almost achieves ML performance and outperforms common SIC by approximately $6 \mathrm{~dB}$ for BER $=10^{-5}$. As strong correlation increases the probability of ill-conditioned channel matrices, the performance of all detectors degrades for $\rho=0.6$. The common ZF-SIC is not able to achieve acceptable performance and also the common MMSE-SIC results in a large performance degradation. The LR-aided detection schemes behave more robust to correlation, as expected by Fig. 2. The LRMMSE-SIC still achieves almost ML performance and obtains an improvement of approximately $11 \mathrm{~dB}$ in comparison to common MMSE-SIC and yields a gain of $4 \mathrm{~dB}$ to the LR-ZF- 
SIC scheme. Consequently, the LR-based MMSE-SIC scheme behaves very robust with respect to spatial correlation and achieves almost ML performance with small computational complexity, as indicated by Fig. 4 and Fig. 5. Due to the benefit of the MMSE solution we will disregard the ZF solution for the remainder of this paper.

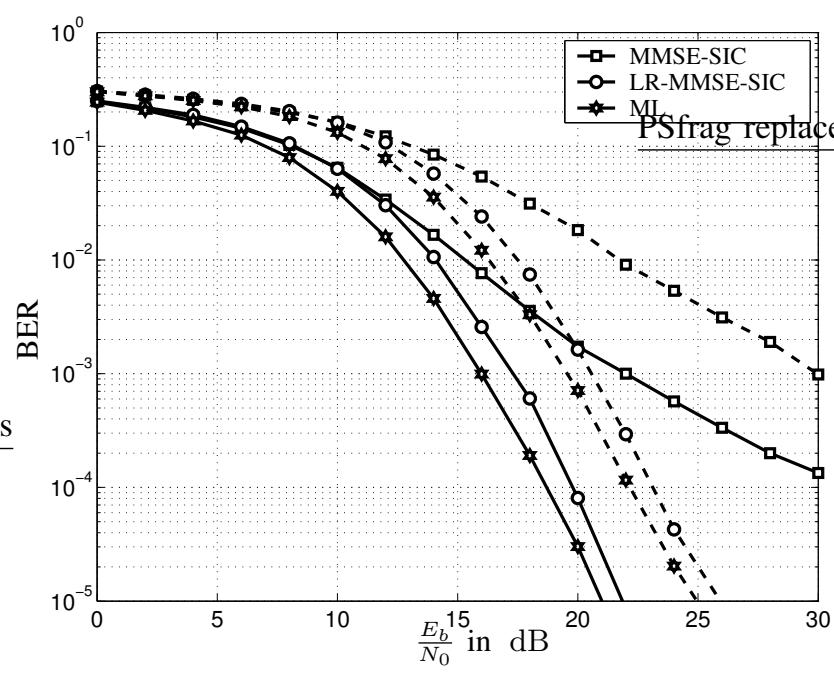

Fig. 7. BER of common and LR-aided MMSE-SIC in a uncorrelated (continuous lines, $\rho=0.0$ ) and correlated (dashed lines, $\rho=0.6$ ) system with $n_{T}=n_{R}=4$ antennas and 16-QAM modulation.

The BERs for the same antenna configuration but with 16QAM modulation are shown in Fig. 7. Again, LR-MMSE-SIC achieves very good performance results and leads only to a degradation of approximately $0.9 \mathrm{~dB}$ for the uncorrelated as well as for the correlated case. It is worth to note, that the computational effort of LLL does certainly not depend on the modulation index. In contrast, a brute force ML would require the comparison of $M^{n_{T}}=65536$ hypotheses for each receive signal vector and is thereby not feasibly anymore. Of course, ML detection can now only be achieved by sphere detection.

In order to investigate the performance for larger number of antennas, Fig. 8 shows the corresponding simulation results for a system with $n_{T}=n_{R}=6$ antennas. The LR-MMSESIC scheme outperforms the common MMSE-SIC scheme by more than $8 \mathrm{~dB}$ for a BER of $10^{-5}$. Thus, the robustness of the LR-aided detection has been shown for several system constellations. As the LR-aided detection does not incorporate the correlation structure, similar results can also be expected for other correlation models.

\section{SUMMARY AND CONCLUSIONS}

In this paper, we investigated common and lattice-reductionaided detection schemes for multiple antenna systems with uncorrelated and correlated channels. The combination of LRMMSE and SIC achieves almost maximum-likelihood performance over the whole range of condition numbers and thereby also for strong spatial correlation. The new scheme clearly outperforms common detectors with low computational complexity.

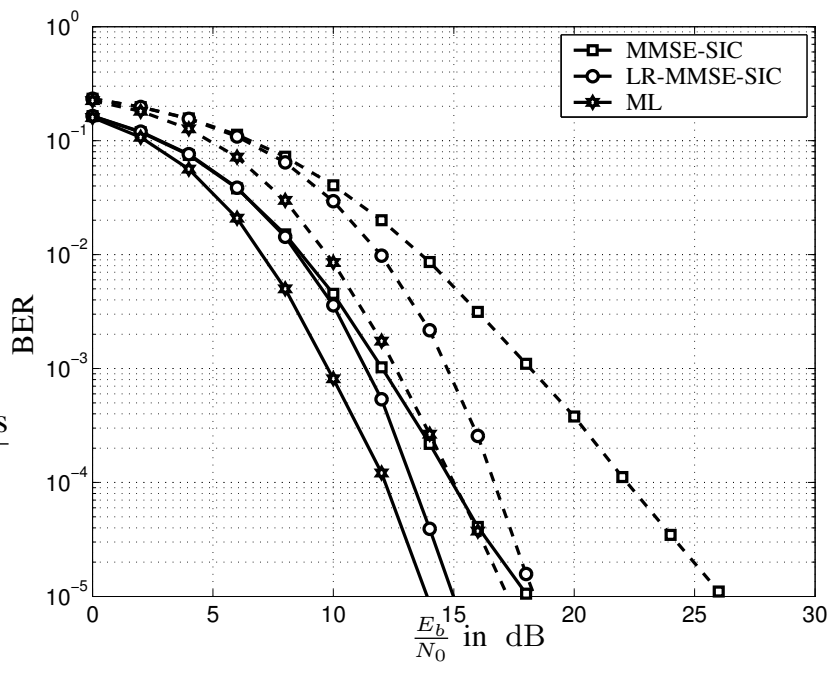

Fig. 8. BER of common and LR-aided MMSE-SIC in a uncorrelated (continuous lines, $\rho=0.0$ ) and correlated (dashed lines, $\rho=0.6$ ) system with $n_{T}=n_{R}=6$ antennas and 4-QAM modulation.

\section{REFERENCES}

[1] H. Yao and G. Wornell, "Lattice-Reduction-Aided Detectors for MIMO Communication Systems," in IEEE Proc. Global Communications Conference (GLOBECOM), Taipei, Taiwan, November 17-21 2002.

[2] W. Mow, "Universal Lattice Decoding: Principle and Recent Advances," Wireless Communications and Mobile Computing, Special Issue on Coding and Its Applications in Wireless CDMA Systems, vol. 3, no. 5, pp. 553-569, August 2003.

[3] C. Windpassinger and R. F. H. Fischer, "Low-Complexity NearMaximum-Likelihood Detection and Precoding for MIMO Systems using Lattice Reduction," in IEEE Proc. Information Theory Workshop (ITW), Paris, France, March 2003.

[4] D. Wübben, R. Böhnke, V. Kühn, and K. D. Kammeyer, "MMSE-Based Lattice Reduction for Near-ML Detection of MIMO Systems," in Proc. ITG Workshop on Smart Antennas, Munich, Germany, March 2004.

[5] _ "Near-Maximum-Likelihood Detection of MIMO Systems using MMSE-Based Lattice Reduction," in IEEE Proc. International Conference on Communications (ICC), Paris, France, June 2004.

[6] J. P. Kermoal, L. Schumacher, K. Ingemann, and P. E. Mogensen, "A Stochastic MIMO Radio Channel Model With Experimental Validation," IEEE Journal on Selected Areas in Commununications, vol. 20, no. 6, pp. 1211-1226, August 2002.

[7] A. van Zelst and J. Hammerschmidt, "A Single Coefficient Spatial Correlation Model for Multiple-Input Multiple-Output MIMO Radio Channel," in Proc. of URSI General Assembly, Maastricht, Netherlands, August 2002.

[8] U. Fincke and M. Pohst, "Improved Methods for Calculating Vectors of Short Length in a Lattice, Including a Complexity Analysis," Math. Comp, vol. 44, pp. 463-471, 1985.

[9] C. P. Schnorr and M. Euchner, "Lattice Basis Reduction: Improved Practical Alorithms and Solving Subset Sum Problems," Mathematical Programming, vol. 66, pp. 181-191, 1994.

[10] E. Agrell, T. Eriksson, A. Vardy, and K. Zeger, "Closest Point Search in Lattices," IEEE Transactions on Information Theory, vol. 48, no. 8, pp. 2201-2214, August 2002.

[11] R. Böhnke, V. Kühn, and K. D. Kammeyer, "Efficient Near-MaximumLikelihood Decoding of Multistratum Space-Time Codes," in IEEE Proc. Vehicular Technology Conference (VTC), Los Angeles, CA, USA, September 2004.

[12] A. K. Lenstra, H. W. Lenstra, and L. Lovász, "Factoring Polynomials with Rational Coefficients," Math. Ann, vol. 261, pp. 515-534, 1982.

[13] D. Wübben, R. Böhnke, V. Kühn, and K. D. Kammeyer, "MMSE Extension of V-BLAST based on Sorted QR Decomposition," in IEEE Proc. Vehicular Technology Conference (VTC), Orlando, Florida, USA, Ocober 2003. 